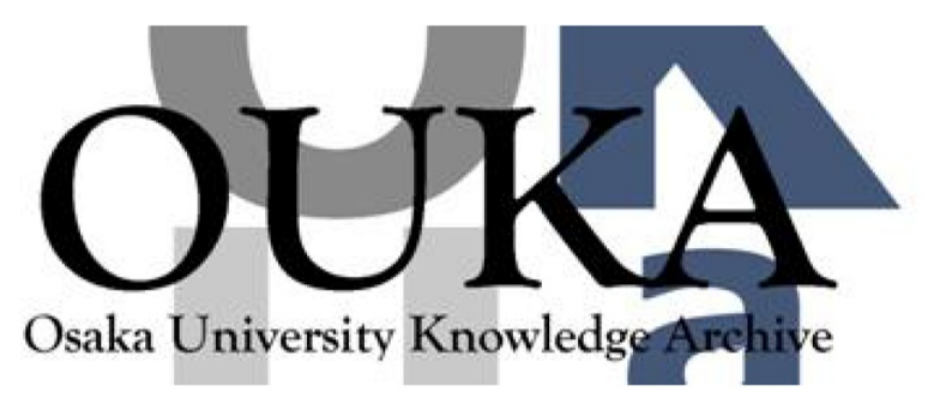

\begin{tabular}{|c|c|}
\hline Title & $\begin{array}{l}\text { Generation of narrowband elastic waves with a } \\
\text { fiber laser and its application to the imaging } \\
\text { of defects in a plate }\end{array}$ \\
\hline Author (s) & Hayashi, Takahiro; Ishihara, Ken \\
\hline Citation & ULtrasonics. 77 p. 47-p. 53 \\
\hline Issue Date & $2017-05$ \\
\hline oaire:version & AM \\
\hline URL & https://hdl. handle. net/11094/84486 \\
\hline rights & $\begin{array}{l}\text { ( } 2017 \text { Elsevier B.V. This manuscript version is } \\
\text { made avai lable under the Creative Commons } \\
\text { Attribut ion-NonCommercial-NoDerivatives } 4.0 \\
\text { International License. }\end{array}$ \\
\hline Note & \\
\hline
\end{tabular}

Osaka University Knowledge Archive : OUKA

https://ir. Library. osaka-u. ac. jp/

Osaka University 


\title{
Generation of narrowband elastic waves with a fiber laser and its application to the imaging of defects in a plate
}

Takahiro Hayashi* and Ken Ishihara**

*Graduate School of Engineering, Kyoto University, Kyoto, 615-8540, Japan

** Faculty of Engineering, Kyoto University, Kyoto, 615-8540, Japan

\begin{abstract}
Pulsed laser equipment can be used to generate elastic waves through the instantaneous reaction of thermal expansion or ablation of the material; however, we cannot control the waveform generated by the laser in the same manner that we can when piezoelectric transducers are used as exciters. This study investigates the generation of narrowband tone-burst waves using a fiber laser of the type that is widely used in laser beam machining. Fiber lasers can emit laser pulses with a high repetition rate on the order of $\mathrm{MHz}$, and the laser pulses can be modulated to a burst train by external signals. As a consequence of the burst laser emission, a narrowband tone-burst elastic wave is generated. We experimentally confirmed that the elastic waves agreed well with the modulation signals in time domain waveforms and their frequency spectra, and that waveforms can be controlled by the generation technique. We also apply the generation technique to defect imaging with a scanning laser source. In the experiments, with small laser emission energy, we were not able to obtain defect images from the signal amplitude due to low signal-to-noise ratio, whereas using frequency spectrum peaks of the tone-burst signals gave clear defect images, which indicates that the signal-to-noise ratio is improved in the frequency domain by using this technique for the generation of narrowband elastic waves. Moreover, even for defect imaging at a single receiving point, defect images were enhanced by taking an average of distributions of frequency spectrum peaks at different frequencies.
\end{abstract}


Keywords: Non-destructive evaluation, Guided waves, Laser ultrasonics, Narrowband waves, Scanning laser source technique

Contact author: Takahiro Hayashi: Graduate School of Engineering, Kyoto University C3 Kyotodaigaku-katsura, Nishikyo-ku, Kyoto, Japan

+81-75-383-3797 (phone\&fax)

e-mail: hayashi@kuaero.kyoto-u.ac.jp 


\section{Introduction}

Laser ultrasonics, whereby ultrasonic waves are generated and detected by using a laser, has been widely studied as a non-contact nondestructive evaluation (NDE) technique [1]. Generally, elastic waves are generated by ablation or by thermoelastic expansion in laser pulse emission, and are detected, using a continuous or long pulse laser, by measuring the change in light intensity when scattered light from the surface of a test object interferes with a reference beam.

The non-contact ultrasonic NDE has already been applied to manufacturing in production lines and high-temperature environments; large structures such as tunnels, bridges, and buildings; and imaging systems in which the internal status of an object is visualized from a large number of signals recorded by scanning the laser or the object. However, laser ultrasonics has not become a viable alternative to ultrasonic measurements based on piezoelectric transducers because of technical limitations of the laser method. For example, in comparison to elastic wave generation with piezoelectric transducers, the laser technique generally has several weak points, such as: 1) Ultrasonic signals generated by the laser technique are small. 2) Laser techniques cannot control the directivities of ultrasonic beams, for example, oblique incidence, the way that an angle beam transducer can. 3) The laser technique can generate only broadband pulse waves while piezoelectric transducers can be made to produce arbitrary waveforms by controlling the input electric signals.

To overcome these weak points, a wide variety of laser ultrasonic generation techniques have been proposed. Wagner et al. developed the generation technique of narrowband elastic wave by high frequency repetition of Q-switching of Nd: YAG laser [2]. Pierce et al. experimentally confirmed generation of narrow band elastic waves by modulating long-pulse Nd: YAG laser using an acousto-optic modulator (Bragg cell) [3]. 
Spatial modulation of laser source also has been published as another approach to enhance laser-generated ultrasonic. McKie et al. experimentally verified the narrow band ultrasonic generation by spatial modulation with a lenticular array [4]. Yamanaka et al. showed that a selective mode of Lamb waves can be enhanced by scanning long-pulse YAG laser at a phase velocity of the mode [5]. Chenu et al. presented that a single giant Rayleigh wave can be generated using multi laser pulses with an appropriate time delay [6]. Noroy et al. [7] and Murray et al. [8] used plural number of laser equipment to establish laser ultrasonic phased array. Yang et al. also developed laser ultrasonic phased array with optical fibers having different lengths to give appropriate time delays [9]. More simple technique of spatial modulation is to use a masking plate with slits whose inter-distance matches to wavelength of a desired ultrasonic mode. Baldwin et al. verified the generation of a narrow band Lamb wave by irradiating expanded laser beam to the mask [10].

These pioneering ultrasonic generations have been applied to material non-destructive evaluation [11 - 13]. The authors previously proposed laser generation for narrowband ultrasonic waves, for the purpose of defect imaging with a scanning laser source technique, using high-repetition fiber lasers and investigated the validity of the wave control technique by numerical calculation [14]. The present study experimentally confirms that narrowband elastic waves with different frequencies can be generated by controlling the laser output with external modulation signals and that the elastic wave generation technique is effective for defect imaging with the scanning laser source.

\section{Elastic wave generation by a fiber laser}

\subsection{Outline of the technique}

Pulsed lasers such as $\mathrm{CO}_{2}$ lasers and Q-switched Nd: YAG lasers have been widely used in ultrasonic generation for non-destructive material evaluation. The laser equipment, which 
can generate a short pulse in the nanosecond range with a high energy of the order of milli-joules per pulse, is also used for laser processing. Although broadband pulse waves having good temporal and spatial resolutions are normally useful for non-destructive material evaluation, we often encounter the problem that a short pulse cannot be distinguished in measurements with a low signal-to-noise ratio (SNR).

Recently, fiber lasers have become useful in laser processing because they improve processing quality owing to their good optical quality compared to $\mathrm{CO}_{2}$ and $\mathrm{Nd}$ : YAG lasers. Although fiber lasers generate smaller pulse energy than conventional pulsed lasers, the large number of laser pulses with a high repetition rate in the $\mathrm{kHz}$ to $\mathrm{MHz}$ range enables the desired processing. In this study, narrowband wave trains, generally called tone-burst waves, are generated using a fiber laser with a high repetition frequency.

In fiber laser equipment, the output can be controlled by external modulation signals. Figure 1 is a schematic example of laser pulses output from the fiber laser in response to external modulation signals. In this study, square waves were used as the modulation signals to switch the laser output; laser pulse burst trains were generated as shown in Fig. 1 with a laser pulse frequency of $210 \mathrm{kHz}$ and an external modulation frequency of a few $\mathrm{kHz}$. In the experiments to be described below, elastic waves corresponding to the external modulation signals were generated by directing the laser pulse trains onto the surface of a metal plate.

\subsection{Experiments of narrowband elastic wave generation}

Figure 2 shows the experimental system used in this study. The fiber laser equipment is an SPI laser G4 50W-S-HS, whose maximum output is $50 \mathrm{~W}$; its repetition frequency while delivering maximum power ranges from $70 \mathrm{kHz}$ to $580 \mathrm{kHz}$; repetition rates up to $1 \mathrm{MHz}$ are also possible at reduced output power. Laser output pulses were controlled by sending 
modulation signals, as shown in Fig. 1, from a function generator to the fiber laser equipment. The laser pulses were directed to the surface of an aluminum alloy test plate after being collimated to a diameter of about $3 \mathrm{~mm}$, and reflected from two mirrors on automatic rotary stages. The laser beam can be positioned at desired points by rotating the stages appropriately. The test plate was $500 \mathrm{~mm} \times 500 \mathrm{~mm} \times 6 \mathrm{~mm}$; artificial defects consisting of 2-mm-wide notches with various depths ranging from $3 \mathrm{~mm}$ to $0.5 \mathrm{~mm}$ were engraved on the surface opposite to the laser emission surface. In the defect imaging experiments described in section 3, only the zigzag artificial defect with the 3-mm depth was used. Piezoelectric disk transducers with a resonant frequency of $6 \mathrm{kHz}$ were attached on the plate with adhesive tape to detect the elastic waves. One piezoelectric transducer was attached just behind the laser aiming point at the center of the plate for detecting the elastic wave clearly, the other four piezoelectric transducers, located near the plate edges on the laser emission surface as shown in Fig. 2, were used for the defect imaging experiments described in the next section. The signals detected by the piezoelectric transducers were amplified by $60 \mathrm{~dB}$ and digitized by $\mathrm{AD}$ converters. Then, bandpass filtering and a fast Fourier transform were applied in a personal computer. Scattered light from the plate was also detected to measure laser output.

First, we confirmed waveforms by aiming the laser at the center of the test plate and detecting elastic waves with the piezoelectric disk just behind the laser spot. The modulation signals from the function generator were square waves in the frequency range from $3 \mathrm{kHz}$ to $10 \mathrm{kHz}$ with a $50 \%$ duty ratio, a tone-burst signal duration of $2 \mathrm{~ms}$, and a repetition interval of $100 \mathrm{~ms}$. The repetition frequency of the laser output was $210 \mathrm{kHz}$, and the output energy per pulse was about $0.24 \mathrm{~mJ}$.

Figure 3(a) shows the laser pulses detected by the photo detector for the $6 \mathrm{kHz}$ modulation signal, and Fig. 3(b) displays the zoomed portion from $0-0.3 \mathrm{~ms}$, showing 
each individual laser pulse. The vertical axis is not calibrated in the figures because the voltage values corresponding to the light intensity were dependent on the angle of the photo detector and the gain of an internal amplifier. However, we can estimate that the pulse peaks in (a) and (b) indicate an output energy per pulse of about $0.24 \mathrm{~mJ}$. These figures show that the appropriate laser beams were emitted by the fiber laser and modulation signals. Elastic waves detected by the piezoelectric transducers were averaged 10 times and filtered through a pass-band of $2-20 \mathrm{kHz}$. Waveforms of the detected signals and their frequency spectra are shown in (c) and (d), respectively. These figures demonstrate that narrowband tone-burst waves follow the external modulation signals of 3 $-10 \mathrm{kHz}$ very faithfully for the first $2 \mathrm{~ms}$. The detected waveforms for 9 and $10 \mathrm{kHz}$ are smaller because the resonant frequency of the piezoelectric transducer is $6 \mathrm{kHz}$. The small waves occurring after $2 \mathrm{~ms}$ are reflections from plate edges and from defects.

\section{Applications to scanning laser source technique}

\subsection{Outline of defect imaging with scanning laser source technique}

This section verifies the validity of the narrowband wave generation by applying it to defect imaging with a scanning laser source technique.

In the field of non-contact elastic wave generation and detection with lasers, elastic waves can be generated relatively easily just by directing the laser beam onto an object; however, elastic wave detection often encounters problems in stable measurements of the light scattered from the surface of an object. In particular, for outdoor structures having rough and inclined surfaces, we often cannot detect scattered light and accordingly cannot measure the elastic waves. Therefore, we have adopted a scanning laser source technique in which only the ultrasonic laser source is scanned, while receiving devices such as ultrasonic transducers are fixed on the material surface [15 - 17], and have 
developed a defect imaging technique for plates using the characteristics of guided wave propagation in plates [18 - 20].

In the experiments reported here, narrowband tone-burst waves were generated by directing modulated laser pulses to the plate; these pulses induced elastic waves that were received at the four piezoelectric transducers $(\mathrm{Ch} .1-4)$ as shown in Fig. 2. The generation laser was scanned over the plate by rotating the two mirrors. Repeating the laser source positioning, laser emission, and measurements of elastic waves gave a large number of waveforms at many scanning points for four channels. Amplitude distributions obtained from the waveforms correspond to defect images in the plate. This is based on the Lamb wave characteristics that we have discussed in previous papers; the amplitude of an A0 mode in the low frequency range becomes larger when the laser beam is directed at a thin defective area, while it becomes smaller for a thick intact area. Taking average of the amplitude distributions from multiple channels gives a clearer defect image by reducing spurious images.

Here we generated $6 \mathrm{kHz}$ tone-burst waves using a modulation signal with a burst duration of $2 \mathrm{~ms}$ as shown before. Received signals filtered by the $2 \mathrm{kHz}-20 \mathrm{kHz}$ bandpass filter with no averaging were recorded for up to $10 \mathrm{~ms}$. In all experiments, the zigzag defect image was obtained by scanning the laser source over the area of $64 \mathrm{~mm} \times 40$ mm behind the zigzag defect (consisting of 3-mm-deep notches) as shown in Fig. 2. In the experimental results shown below, RF signal distributions obtained from the maximum values of signals recorded over intervals up to $10 \mathrm{~ms}$ will be compared, and FFT distributions obtained from peaks of the frequency spectra will also be compared. Hereafter, these distributions will be called an RF distribution or an FFT distribution, respectively, and the averaged image of these distributions will be called an RF defect image or an FFT defect image, respectively. 


\subsection{Defect images by signal amplitude and FFT peak}

Figure 4 (a) - (d) displays the RF distributions obtained from four channels for a $6 \mathrm{kHz}$ tone-burst wave with a modulation frequency that is very close to the resonant frequency of the piezoelectric transducers; the RF defect image in Fig. 4 (e) is the averaged image of (a) - (d). In order to compare this with the FFT defect images to be shown later, another RF defect image is also shown in (f), where the SNR was intentionally made lower by reducing the energy output by 50\%. All distributions in Fig. 4 were normalized to the maximum amplitude of all signals from all channels, and the distributions shown later are also normalized in the same way.

The RF distributions (a) - (d) do not show the zigzag images very clearly because of random noise covering the entire images; this is due to the small SNR in the RF signals. In the RF defect image (e) which is the average of images of (a)-(d), spurious artifacts and random noises were reduced, and the image of the zigzag defect was enhanced. Similar results were obtained in our previous studies $[19,20]$. When we reduced laser output to $50 \%$, the RF defect image (f) did not show the zigzag image because the noise level exceeded the RF signal.

Next, Fig. 5 (a) - (d) show FFT distributions obtained from the spectrum peaks of the RF signals used in Fig. 4. Random noise is significantly reduced in these images compared to Fig. 4. These results indicate that the SNR is enhanced in the frequency domain because the tone-burst wave generation results in distinct peaks of the FFT spectra. Although the FFT distributions Fig. 5(a) - (d) still do not exhibit well-defined zigzag patterns, the FFT defect image Fig. 5(e), which is an average of (a) - (d), does exhibit a very clear zigzag pattern. Even when the laser output is reduced to $50 \%$, the zigzag pattern can be seen in the FFT defect image of Fig. 5(f), while it did not appear at all in the RF defect image of Fig. 4(f). These results clearly show that tone-burst wave generation using 
a fiber laser is effective for elastic wave generation with a laser that generally provides small signal levels.

Next, we discuss defect images obtained for various frequencies of the tone-burst waves. Figure 6 shows the RF defect images for different modulation signals of 3, 5, 7, and $9 \mathrm{kHz}$. Just as in Fig. 4, the RF defect images were obtained from the maximum values of the RF signals over a $10 \mathrm{~ms}$ interval, filtered by a bandpass filter of $2-20 \mathrm{kHz}$ without averaging. Figure 7 presents FFT defect images created by taking frequency spectrum peaks of the RF signals. At all frequencies, the RF defect images in Fig. 6 contain noisy images showing low SNR compared with the FFT defect images in Fig. 7. Particularly in $6(\mathrm{a})$, using $3 \mathrm{kHz}$ modulation frequency that is far removed from the resonant frequency of the piezoelectric transducers, the zigzag pattern is obscured in the RF defect image because of the small SNR, while a clear zigzag pattern can be seen in the FFT defect image of Fig. 7(a).

\subsection{Defect imaging at a signal receiving point}

In the above discussions, the defect images were created by taking an average of the RF or FFT distributions at four different receiving points. However, we can consider many cases where multiple receiving points are not easily prepared. For example, if the receiving devices are laser Doppler vibrometers (LDVs) rather than piezoelectric transducers, multiple receptions by multiple LDVs are not reasonable. Therefore, it is usually highly desirable to have only one receiving point. Here, we investigate the requirements for obtaining a clear image from a single receiving point.

Figures 8 (a) - (d) are FFT distributions created by RF signals detected at Ch. 4 when tone-burst waves at four different frequencies are employed. Noisy dots can be seen in Fig. 8 (a), showing a low SNR, while spurious images and lack of zigzag patterns are 
seen in (c) and (d). When we take the average of these images (a) - (d), a more distinct zigzag pattern can be obtained as shown in Fig. 8(e). Just as in the case of the averaged image of different receiving positions, an averaging technique was utilized to enhance the images so that the characteristics of the zigzag pattern could be obtained at the same positions for all frequencies while the spurious images are located at different areas for different frequencies.

\section{Conclusions}

This study demonstrated the generation of narrowband tone-burst waves using a fiber laser that can emit laser pulses with extremely high repetition rates while the laser emission is controlled by external modulation signals. First, we confirmed that tone-burst waves can be generated by using modulation signals from $3 \mathrm{kHz}$ to $10 \mathrm{kHz}$. Applying the tone-burst wave generation technique to defect imaging with the scanning laser source, defect images were created clearly using Fourier spectrum peaks of the tone-burst signals. Moreover, even for defect imaging at a single receiving point, defect images were enhanced by taking an average of FFT distributions at different frequencies.

\section{Acknowledgments}

The authors would like to thank Professor Shiro Biwa for his insightful comments and continuing support. This work was supported by JSPS KAKENHI Grant Number 26282094.

\section{References}

[1] C.B. Scruby and L.E. Drain, Laser Ultrasonics: Techniques andApplications (Adam Hilger, Bristol, Philadelphia, and NewYork, 1990).

[2] J.W. Wagner, J.B. Deaton, J.B. Spicer, Generation of ultrasound by repetitively 
Q-switching a pulsed Nd:YAG laser., Appl. Opt. 27 (1988) 4696-700.

[3] R. Pierce, C. Ume, J. Jarzynski, G.W. Woodruff, Temporal modulation of a laser source for the generation of ultrasonic waves, 33 (1995) 133-137.

[4] A.D.W. Mckie, J.W. Wagner, J.B. Spicer, C.M. Penney, Laser generation ultrasound of narrow-band and directed ultrasound, Ultrasonics. 27 (1989) 323-330.

[5] K. Yamanaka, Selective scanning excitation of single-mode of a laser beam acoustic waves by phase velocity, (1991) 1591-1593.

[6] C. Chenu, M.-H. Noroy, D. Royer, Giant surface acoustic waves generated by a multiple beam laser: Application to the detection of surface breaking slots, Appl. Phys. Lett. 65 (1994) 1091.

[7] M.H. Noroy, D. Royer, M. Fink, The laser-generated ultrasonic phased array: analysis and experiments, J. Acoust. Soc. Am. 94 (1993) 1934-1943.

[8] T.W. Murray, J.B. Deaton Jr., J.W. Wagner, Experimental evaluation of enhanced generation of ultrasonic waves using an array of laser sources, Ultrasonics. 34 (1996) 6977.

[9] J. Yang, N. DeRidder, C. Ume, J. Jarzynski, Non-contact optical fibre phased array generation of ultrasound for non-destructive evaluation of materials and processes, Ultrasonics. 31 (1993) 387-394.

[10] K. Baldwin, T. Berndt, M. Ehrlich, Narrowband laser generation/air-coupled detection: ultrasonic system for on-line process control of composites, Ultrasonics. 37 (1999) 329-34.

[11] R.E. Green, Non-contact ultrasonic techniques, Ultrasonics. 42 (2004) 9-16.

[12] F. Lanza Di Scalea, R.E. Green, A hybrid non-contact ultrasonic system for sensing bond quality in tow-placed thermoplastic composites, J. Compos. Mater. 16 (2014) 928-940. 
[13] H. Kim, K. Jhang, M. Shin, J. Kim, A noncontact NDE method using a laser generated focused-Lamb wave with enhanced defect-detection ability and spatial resolution, NDT E Int. 39 (2006) 312-319.

[14] T. Hayashi, K. Yamaguchi, S. Biwa, Numerical analysis of narrow band ultrasonic wave generation with high repetition pulse laser and laser scanning, J. Phys. Conf. Ser. 520 (2014) 012024.

[15] Y. Sohn, S. Krishnaswamy, Mass spring lattice modeling of the scanning laser source technique, Ultrasonics. 39 (2002) 543-551.

[16] Y. Sohn, S. Krishnaswamy, A near-field scanning laser source technique and a microcantilever ultrasound receiver for detection of surface-breaking defects, Meas. Sci. Technol. 17 (2006) 809-818.

[17] J. Takatsubo, B. Wang, H. Tsuda, N. Toyama, Generation laser scanning method for the visualization of ultrasounds propagating on a 3-D object with an arbitrary shape, J. Solid Mech. Mater. Eng. 1 (2007) 1405-1411.

[18] T. Hayashi, M. Murase, M.N. Salim, Rapid thickness measurements using guided waves from a scanning laser source., J. Acoust. Soc. Am. 126 (2009) 1101-1106.

[19] T. Hayashi, M. Murase, T. Kitayama, Frequency dependence of images in scanning laser source technique for a plate., Ultrasonics. 52 (2012) 636-42.

[20] T. Hayashi, M. Murase, N. Ogura, T. Kitayama, Imaging Defects in a Plate with Full Non-Contact Scanning Laser Source Technique, Mater. Trans. 55 (2014) 1045-1050. 


\section{Figure Captions}

Fig. 1. Schematic representation of the output laser pulse from a fiber laser equipment, and the external modulation signal used to control the laser output.

Fig. 2. Experimental set-up and an aluminum alloy test plate used in this study

Fig. 3. Fig. 3 Laser pulses emitted and elastic waves generated.
(a) Light intensity transition.
(b) Zoomed figure of (a).
(c) Waves detected by the piezoelectric transducer
(d) Frequency spectra of (c).

Fig. 4. Amplitude distributions (RF distributions) and averaged images (RF defect images) for $6 \mathrm{kHz}$ incidence.
(a) Ch. 1
(b) Ch. 2
(c) Ch. 3
(d) Ch. 4
(e) Average
(f) Average, $50 \%$ energy

Fig. 5. FFT distributions and averaged images (FFT defect images) for $6 \mathrm{kHz}$ incidence.
(a) Ch. 1
(b) Ch. 2
(c) Ch. 3
(d) Ch. 4
(e) Average
(f) Average, 50\% energy

Fig. 6. RF defect images at different frequencies.
(a) $3 \mathrm{kHz}$
(b) $5 \mathrm{kHz}$
(c) $7 \mathrm{kHz}$
(d) $9 \mathrm{kHz}$

Fig. 7. FFT defect images at different frequencies.
(a) $3 \mathrm{kHz}$
(b) $5 \mathrm{kHz}$
(c) $7 \mathrm{kHz}$
(d) $9 \mathrm{kHz}$ 
Fig. 8. FFT distributions at different frequencies at a single receiving point (Ch. 4) and their averaged image.
(a) $3 \mathrm{kHz}$
(b) $5 \mathrm{kHz}$
(c) $7 \mathrm{kHz}$
(d) $9 \mathrm{kHz}$
(e) Average 


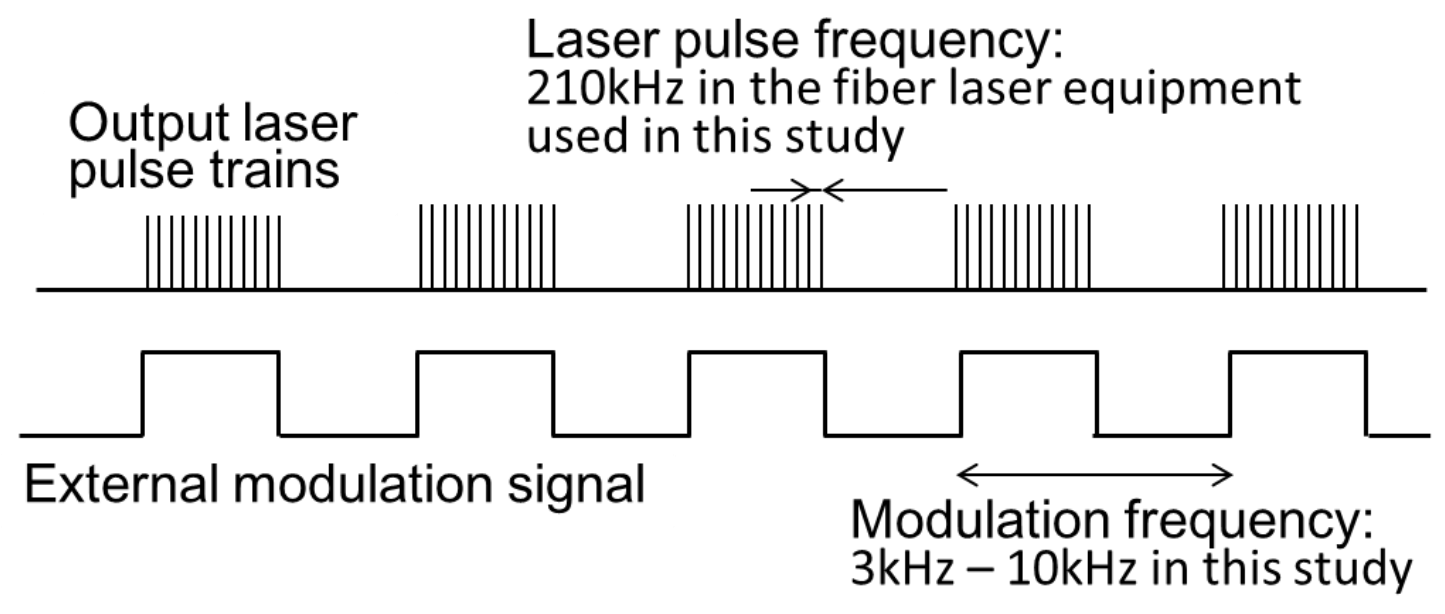

Fig.1. Schematic representation of the output laser pulse from a fiber laser equipment, and the external modulation signal used to control the laser output. 


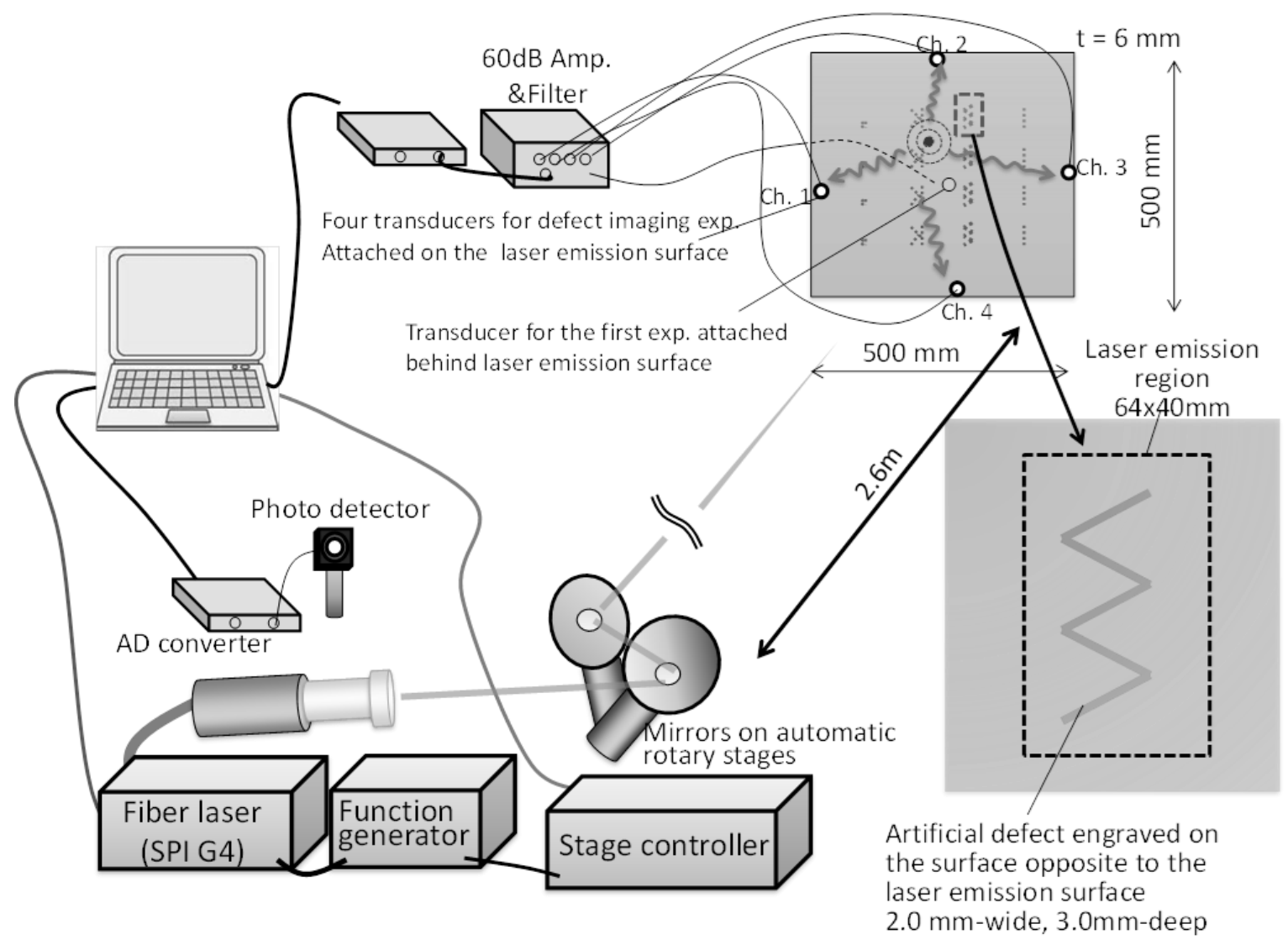

Fig. 2. Experimental set-up and an aluminum alloy test plate used in this study 


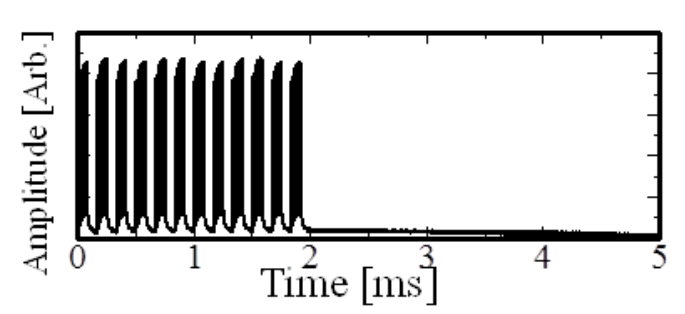

(a)

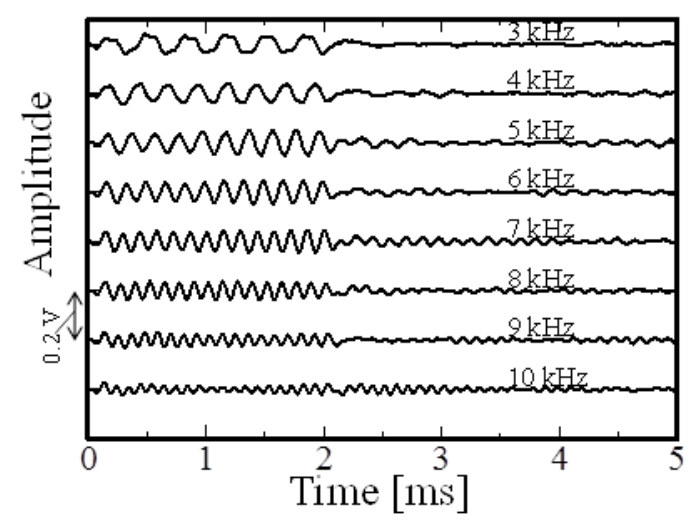

(c)

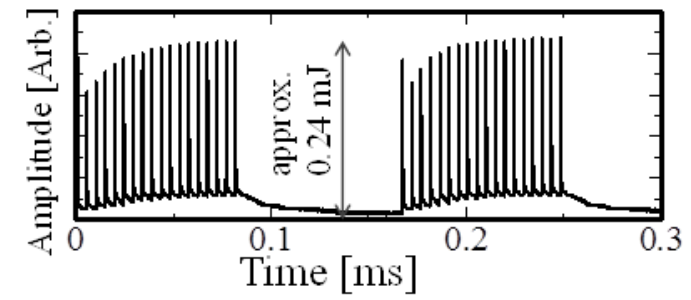

(b)

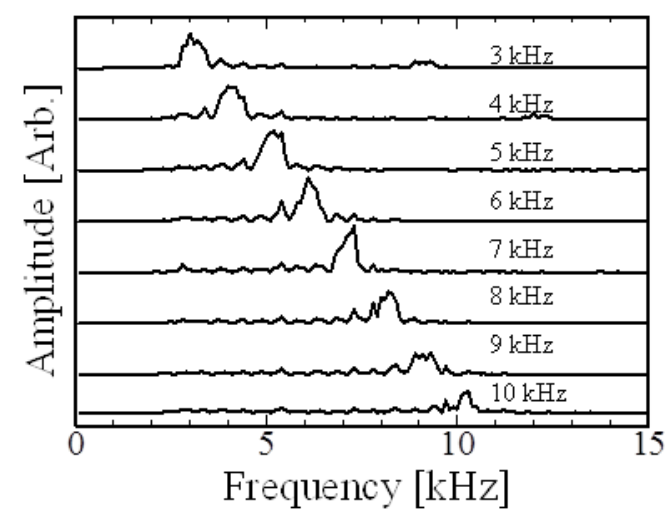

(d)

Fig. 3 Laser pulses emitted and elastic waves generated.

(a) Light intensity transition, (b) Zoomed figure of (a)

(c) Waves detected by the piezoelectric transducer, (d) Frequency spectra of (c). 


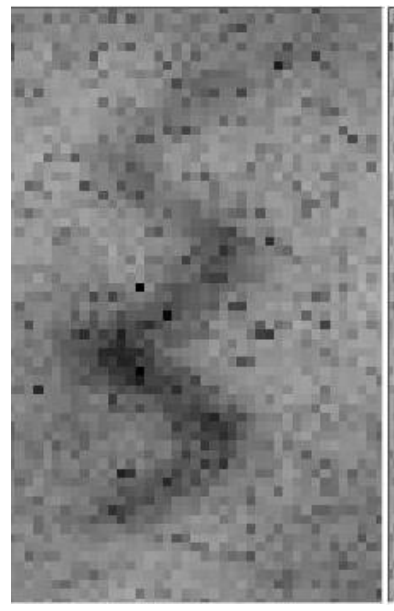

(a) Ch. 1

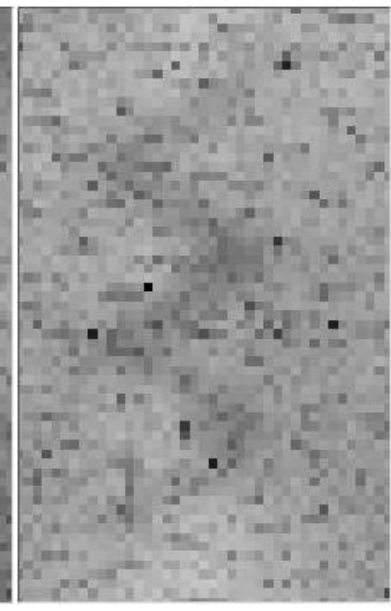

(b) Ch. 2

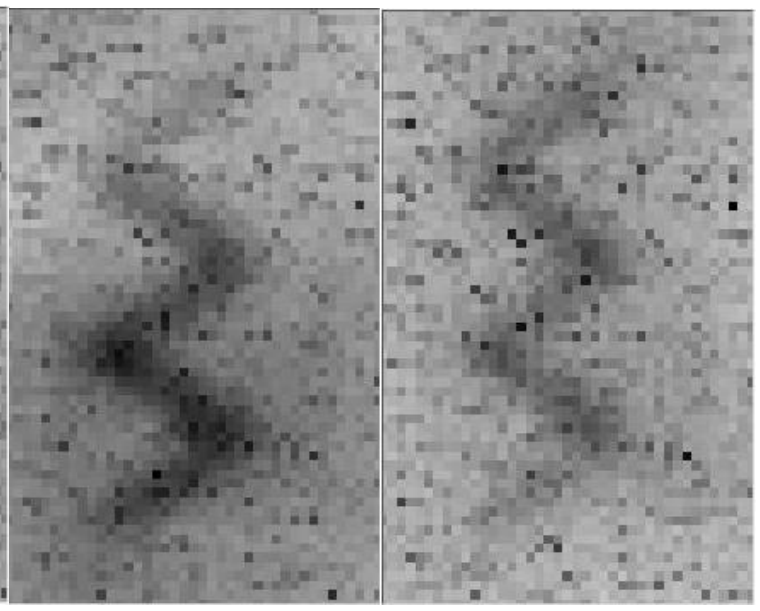

(c) Ch. 3

(d) Ch. 4

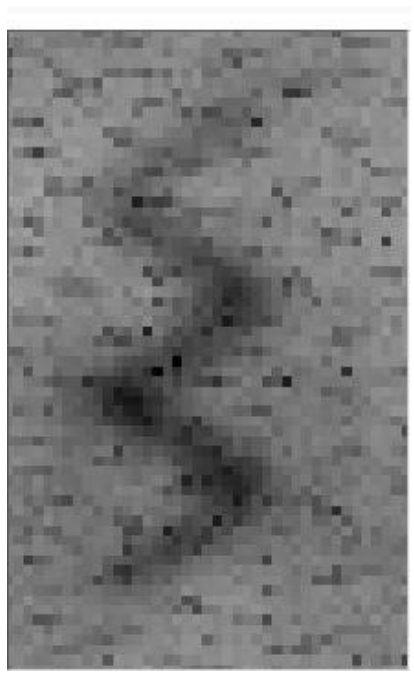

(e) Average

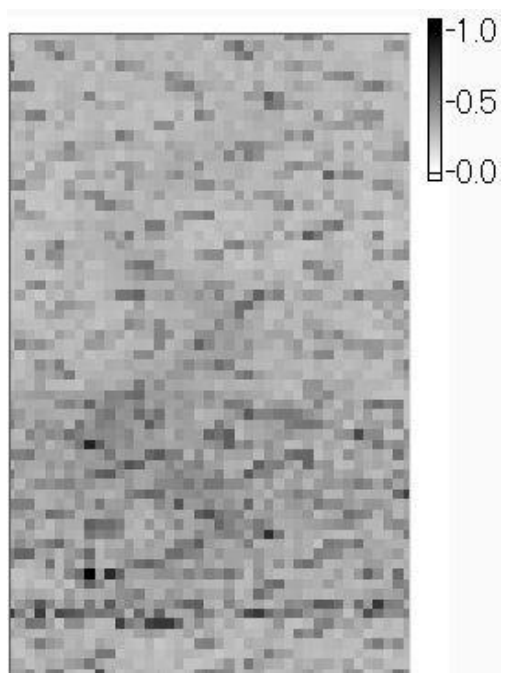

(f) Average, $50 \%$ energy

Fig. 4. Amplitude distributions (RF distributions) and averaged images (RF defect images) for $6 \mathrm{kHz}$ incidence. 


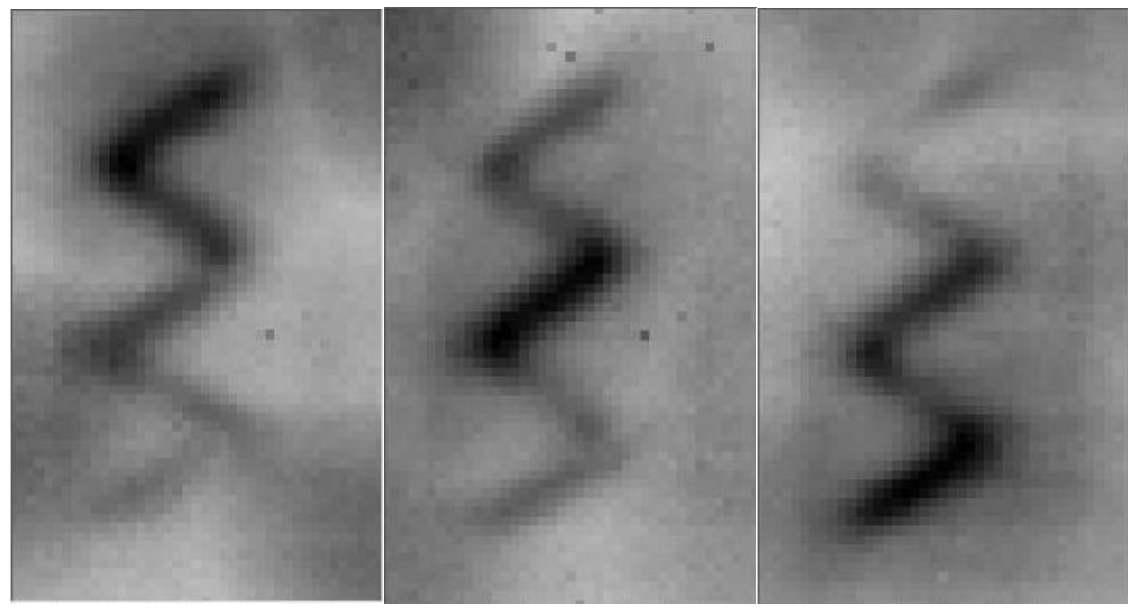

(a) Ch. 1 (b) Ch. 2

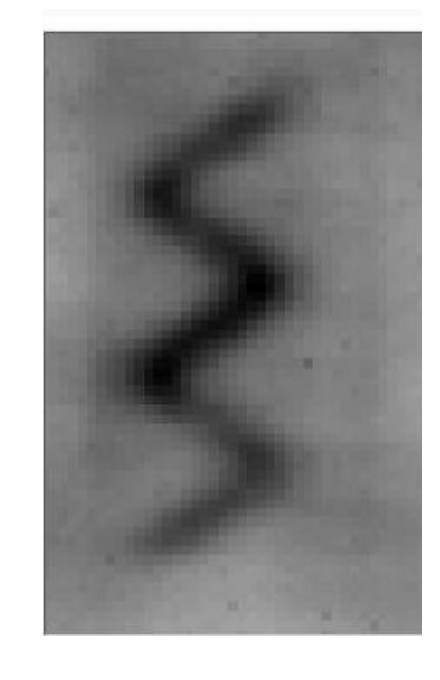

(e) Average

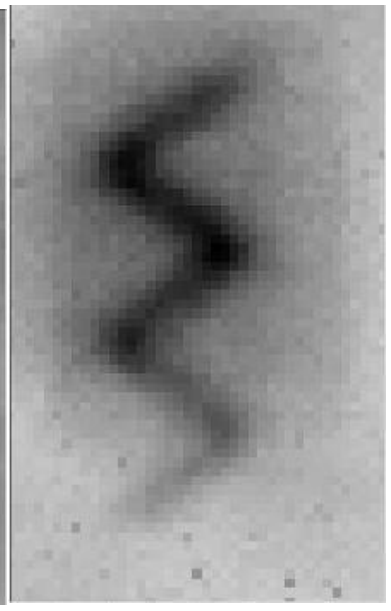

(c) Ch. 3

(d) Ch. 4

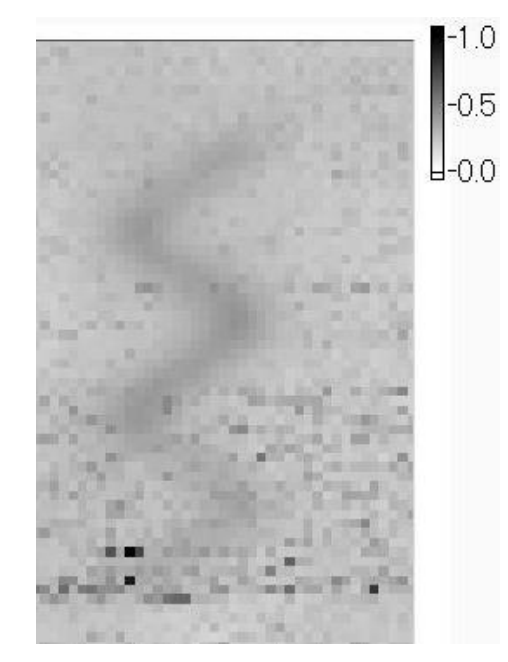

(f) Average, $50 \%$ energy

Fig. 5. FFT distributions and averaged images (FFT defect images) for $6 \mathrm{kHz}$ incidence. 


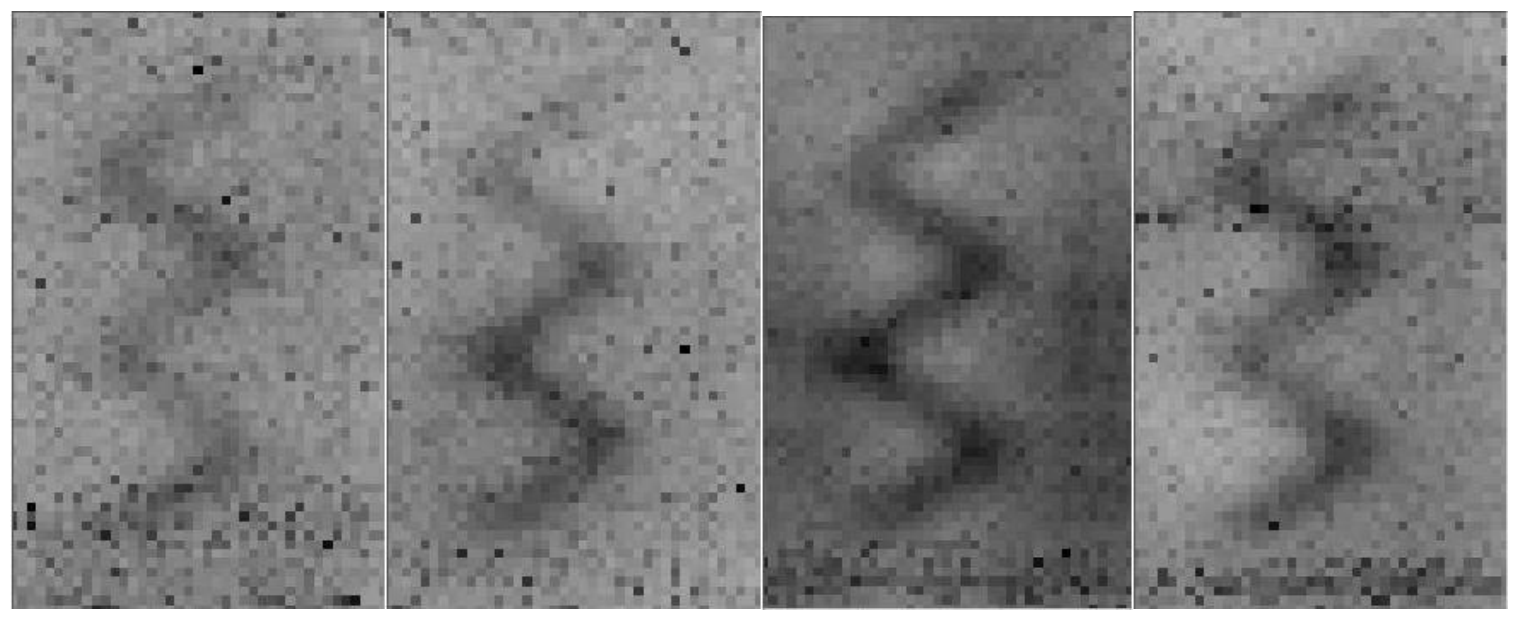
(a) $3 \mathrm{kHz}$
(b) $5 \mathrm{kHz}$
(c) $7 \mathrm{kHz}$
(d) $9 \mathrm{kHz}$

Fig. 6. RF defect images at different frequencies. 


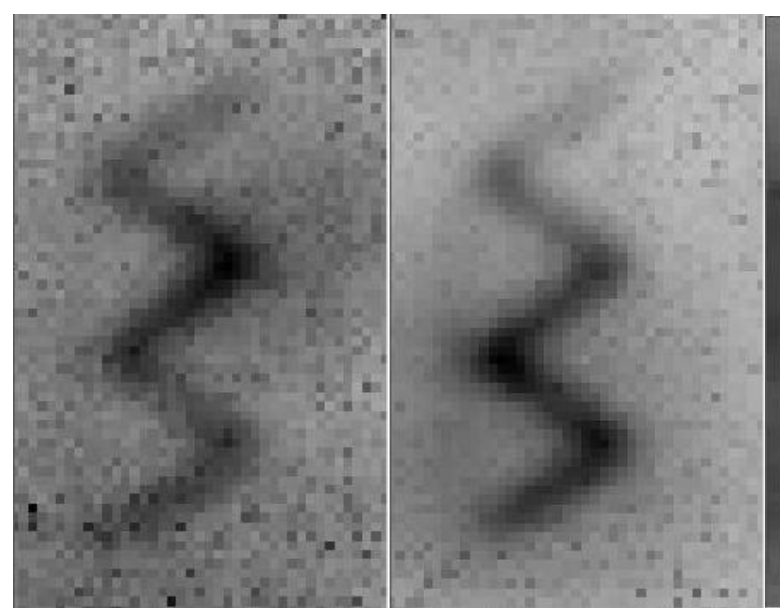

(a) $3 \mathrm{kHz}$

(b) $5 \mathrm{kHz}$

(c) $7 \mathrm{kHz}$

(d) $9 \mathrm{kHz}$

Fig. 7. FFT defect images at different frequencies. 


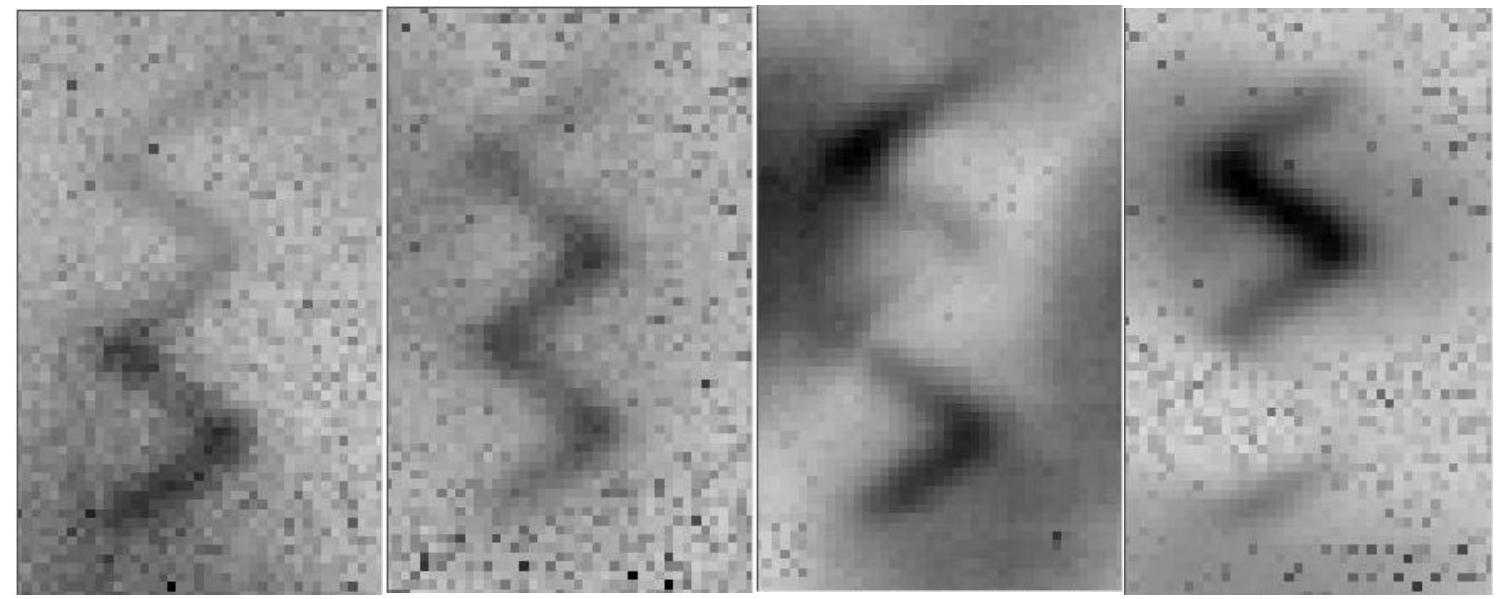

(a) $3 \mathrm{kHz}$

(b) $5 \mathrm{kHz}$

(c) $7 \mathrm{kHz}$

(d) $9 \mathrm{kHz}$

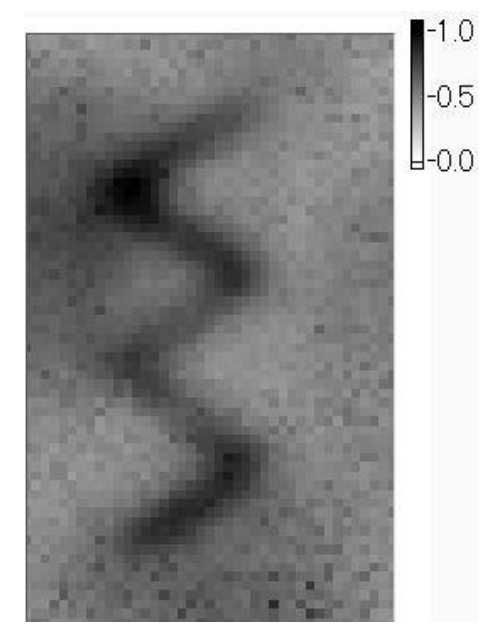

(e) Average

Fig. 8. FFT distributions at different frequencies at a single receiving point (Ch. 4) and their averaged image. 